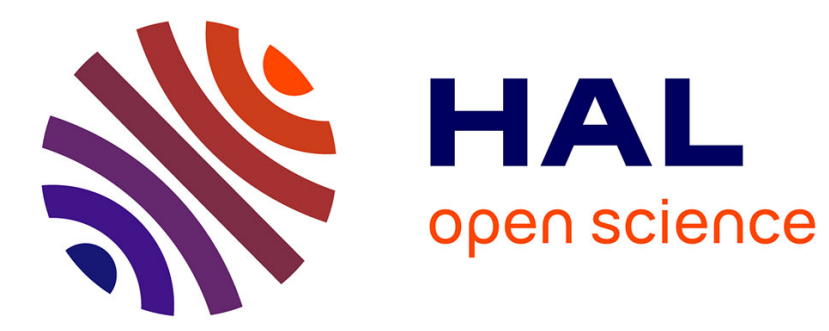

\title{
Structure of Citrus pectins and viscometric study of their solution properties
}

\author{
Monique Axelos M.A.V., J.F. Thibault, J. Lefebvre
}

\section{To cite this version:}

Monique Axelos M.A.V., J.F. Thibault, J. Lefebvre. Structure of Citrus pectins and viscometric study of their solution properties. International Journal of Biological Macromolecules, 1989, 11 (3), pp.186-191. 10.1016/0141-8130(89)90066-4 . hal-02727026

\section{HAL Id: hal-02727026 \\ https://hal.inrae.fr/hal-02727026}

Submitted on 2 Jun 2020

HAL is a multi-disciplinary open access archive for the deposit and dissemination of scientific research documents, whether they are published or not. The documents may come from teaching and research institutions in France or abroad, or from public or private research centers.
L'archive ouverte pluridisciplinaire HAL, est destinée au dépôt et à la diffusion de documents scientifiques de niveau recherche, publiés ou non, émanant des établissements d'enseignement et de recherche français ou étrangers, des laboratoires publics ou privés. 
 properties
}

\author{
M. A. V. Axelos, J. F. Thibault* and J. Lefebvre \\ INRA, Laboratoire de Physicochimie des Macromolécules, BP 527, 44026 Nantes Cédex 03, France \\ (Received 16 October 1988; revised 26 January 1989)
}

\begin{abstract}
Citrus pectins with degrees of methylation between 30 and $72 \%$ were carefully characterized in order to determine their charge density and molecular weight distribution, the content in galacturonic acid and in neutral sugars, the degree of methylation and acetylation. Using enzymic degradation it has been found that pectin molecules consist mainly of long homogalacturonan regions with some regions of neutral sugars as side chains attached on rhamnose residues. The viscometric behaviour of the different samples indicates that $0.1 \mathrm{M} \mathrm{NaCl}$, at $25^{\circ} \mathrm{C}$, is a good solvent of sodium pectinates. From the evolution of the Huggins parameter, it appears that pectins with $50 \%$ of methylated galacturonic groups exhibit a maximum flexibility. A Mark-Houwink exponent of 0.8 has been found in good agreement with theoretical predictions for flexible polymers in a good solvent.
\end{abstract} Keywords: Ion-exchange chromatography; gel permeation chromatography; enzymic degradation; viscosity; polymer
flexibility; Mark-Houwink exponent

\section{Introduction}

Pectins are a major structural polysaccharide component of the primary cell walls of plants. Extracted mainly from apple pomace and citrus peels, they are used in the food industry as gelling agents ${ }^{1}$. Basically, pectin molecules consist of linearly connected homogalacturonans interrupted by a few rhamnogalacturonan segments carrying neutral sugars as side chains, typically galactose and arabinose ${ }^{2}$. In native pectins, about $70 \%$ of the carboxyl groups are methyl-esterified; these 'high-methoxyl' pectins form gels in an acidic medium at high concentrations of sucrose. Controlled de-esterification yields pectins with lower ester contents; these 'low-methoxyl' pectins, with degree of methylation. (DM) less than $45 \%$, gel in the presence of calcium ions.

The change in the DM gives rise to a large chemical diversity of pectins which leads to very different solution behaviours. Several reports ${ }^{3.4}$ suggest that the size of the pectins in aqueous solution at neutral $\mathrm{pH}$ and constant ionic strength depends on the balance between charge-charge repulsions, hydrogen bonding and hydrophobic interactions. Among the numerous investigations on the DM dependence of the conformation, some contradictory conclusions are found ${ }^{5}$ nevertheless. The main experimental observation is that pectins aggregate in solution. This aggregation seems to be the major problem in the determination of the size of the polymer by methods such as gel chromatography or light scattering which are very sensitive to the presence of aggregates. After ultracentrifugation or filtration of the solutions, they may lead to different results especially for the Mark-Houwink exponent ${ }^{5,6}$. Another important reason

* INRA, Laboratoire de Biochimic et Technologie des Glucides, BP 527, 44026 Nantes Cédex 03, France.

0141-8130/89/030186-06\$03.00

(C) 1989 Butterworth \& Co. (Publishers) Ltd

186 Int. J. Biol. Macromol., 1989, Vol 11, June for this lack of agreement may be the differences in the chemical composition and/or structure of the native pectins and of the fractions obtained by gel permeation chromatography; in the literature very little attention has been paid to this last point.

In the present work, we report on a complete chemical characterization of pectin samples followed by a systematic investigation of the viscosity in $0.1 \mathrm{M} \mathrm{NaCl}$ solution over a large range of concentrations extending from the dilute to the concentrated regimes. This rheological method presents the advantage that it is not perturbed by a small amount of aggregates. We will discuss the dependence of viscosity properties on concentration in terms of solvent power and polymer chain flexibility. An attempt will be made to determine the exponent of the Mark-Houwink equation from rheological data alone.

\section{Materials and methods \\ Preparation of the samples}

Purification. Five g of a commercial HM citrus pectin $\left(P_{72}\right)$ from Genu Pectin (type X-6954 from Copenhagen Pectin Factory) were dissolved in 1 litre of distilled water at room temperature and purified by precipitation with $200 \mathrm{ml}$ of $7 \%(\mathrm{w} / \mathrm{v})$ cupric acetate solution?

The precipitate was collected on a porous glass filter under reduced pressure and extensively washed with distilled water and then dissolved in 1 litre of $2.5 \%(\mathrm{w} / \mathrm{v})$ EDTA disodium salt by stirring 1 night at $4{ }^{\circ} \mathrm{C}$. The resulting solution was dialysed against distilled water until the conductivity was less than $10 \mu \mathrm{S}$ and freeze-dried.

De-esterification. Samples of pectin with different DM were obtained by partial alkaline de-esterification of the purified pectin ${ }^{8}$. Aqueous solutions of pectins were maintained at $2^{\circ} \mathrm{C}$ in an ice-salt bath and the $\mathrm{pH}$ was 
adjusted to 7 with $1 \mathrm{M} \mathrm{NaOH}$. In order to obtain various $\mathrm{DM}$, calculated amounts of hydroxide were added to the solutions corresponding to the methanol to be removed. After 2 days at $2^{\circ} \mathrm{C}$, the solutions were brought to $\mathrm{pH} 4.5$ with $1 \mathrm{M} \mathrm{HCl}$, then dialysed against distilled water and freeze-dried.

Enzymatic degradation. A highly purified endopolygalacturonase (endoPG; EC 3.2.1.15) ${ }^{9}$ was used. The pectin sample was previously entirely de-esterified in 5 $10^{-2} \mathrm{M} \mathrm{NaOH}$ during $2 \mathrm{~h}$ at $4^{\circ} \mathrm{C}$ and then dialysed against a $0.1 \mathrm{M}$ sodium acetate buffer at $\mathrm{pH} 4.2$. Enzyme reaction conditions were as follows: endoPG, 5 nkat $\mathrm{ml}^{-1}$, and $2 \mathrm{mg} \mathrm{ml}^{-1}$ of the de-esterified pectin sample at $30^{\circ} \mathrm{C}$ for at least $40 \mathrm{~h}$. The extent of degradation was calculated from the increase of the reducing power as determined by the method of Nelson ${ }^{10}$ using galacturonic acid as standard.

\section{Analysis}

Chemical analysis. The anhydrogalacturonic (AGA) content of the pectins was determined by an automated m-hydroxydiphenyl/sulphuric acid assay ${ }^{11}$. DM and degrees of acetylation (DA) were calculated from the amount of the methanol and acetic acid as determined by h.p.l.c. after de-esterification ${ }^{12}$. Individual neutral sugars were analysed by gas chromatography as their alditol $\operatorname{acetates}^{13}$.

Gel chromatography. Ion-exchange chromatography was performed on a DEAE-Sepharose CL-6B (Pharmacia) column $(20 \times 1.6 \mathrm{~cm})$ equilibrated with sodium acetate buffer, pH 4.8, ionic strength $0.05 \mathrm{M}$. Pectin (14 mg), dissolved in the same buffer, was loaded onto the column and the gel was washed with $55 \mathrm{ml}$ of the buffer to remove neutral materials. The bound material was then eluted by a linear sodium acetate gradient at $\mathrm{pH} 4.8$ with ionic strength from 0.05 to $1 \mathrm{M}$. The elution profiles were obtained by analysing the AGA and neutral sugars contents by $\mathrm{m}$-hydroxydiphenyl and orcinol ${ }^{14}$ methods, respectively.

Chromatography on a Sepharose CL-2B column $(97.5 \times 1.5 \mathrm{~cm})$ and on a Sephacryl S-200 column $(79 \times 2.2 \mathrm{~cm})$ were carried out in ascending direction with sodium acetate buffer, $\mathrm{pH}=4$, ionic strength $0.1 \mathrm{M}$, at a flow-rate of $25 \mathrm{ml} \mathrm{h}^{-1}$. Four $\mathrm{mg}$ of pectin samples dissolved in the same buffer were applied to the column and 4-6 $\mathrm{ml}$ fractions were collected and analysed as previously described. Results were expressed as a function of the partition coefficient:

$$
K_{\mathrm{av}}=\left(V_{\mathrm{e}}-V_{\mathrm{o}}\right) /\left(V_{\mathrm{t}}-V_{\mathrm{o}}\right)
$$

where $V_{\mathrm{e}}, V_{0}$ and $V_{1}$ are the elution volume of the fraction, the void volume and the total volume of the column, respectively.

\section{Preparation of the solutions}

The acidic form of the samples was obtained by washing the freeze-dried pectins with $80 \%(\mathrm{v} / \mathrm{v})$ ethanol containing $1 \%(\mathrm{v} / \mathrm{v})$ hydrochloric acid. The precipitate was extensively washed with $60 \%$ ethanol and then dried by washing successively with $98 \%$ ethanol, acetone and ether.

The ionic capacities of the pectic acids were determined by $\mathrm{pH}$-metry and the salt-free sodium pectinate solutions were obtained by exact neutralization with sodium hydroxide.

Polymer concentration was calculated from the AGA content ${ }^{11}$.

\section{Viscometry}

Viscosity measurements were performed with a Lowshear 30 Contraves rheometer over a shear rate range of 0.017 to $128 \mathrm{~s}^{-1}$. The temperature was $25.0 \pm 0.1^{\circ} \mathrm{C}$.

\section{Results and discussion \\ Characteristics of the samples}

The results of the ion-exchange chromatography of the initial pectin $\mathrm{P}_{72}$ (Figure 1) show that $\simeq 40 \%$ of the neutral sugars are not bound to the gel and therefore are not associated to the pectic backbone. After purification, the content in neutral sugars is decreased from $7 \%$ to $4 \%$ (w/w) and ion-exchange chromatography shows that they are all linked to the pectin. The yield of the purification process was $87 \%$. The elution pattern indicates a homogeneous distribution of the charge density within the pectic molecules.

After purification, a galacturonic acid content of $78 \%$, a DM of $72 \%$ and a low DA of $1 \%$ are found. The chemical composition of the crude and the purified $\mathrm{P}_{72}$ pectin is shown in Table 1. Galactose and arabinose account for $89 \%$ (molar ratio) of the total sugars, xylose and glucose appear as very minor constituents. It was found around 1 rhamnose for 94 galacturonic acid units.

Figure 2 shows the elution profile of the purified $\mathrm{P}_{72}$ pectin on Sepharose CL-2B. No peak appears in the void volume. The width of the peak in the fractionation range of the column indicates that the pectins have a large

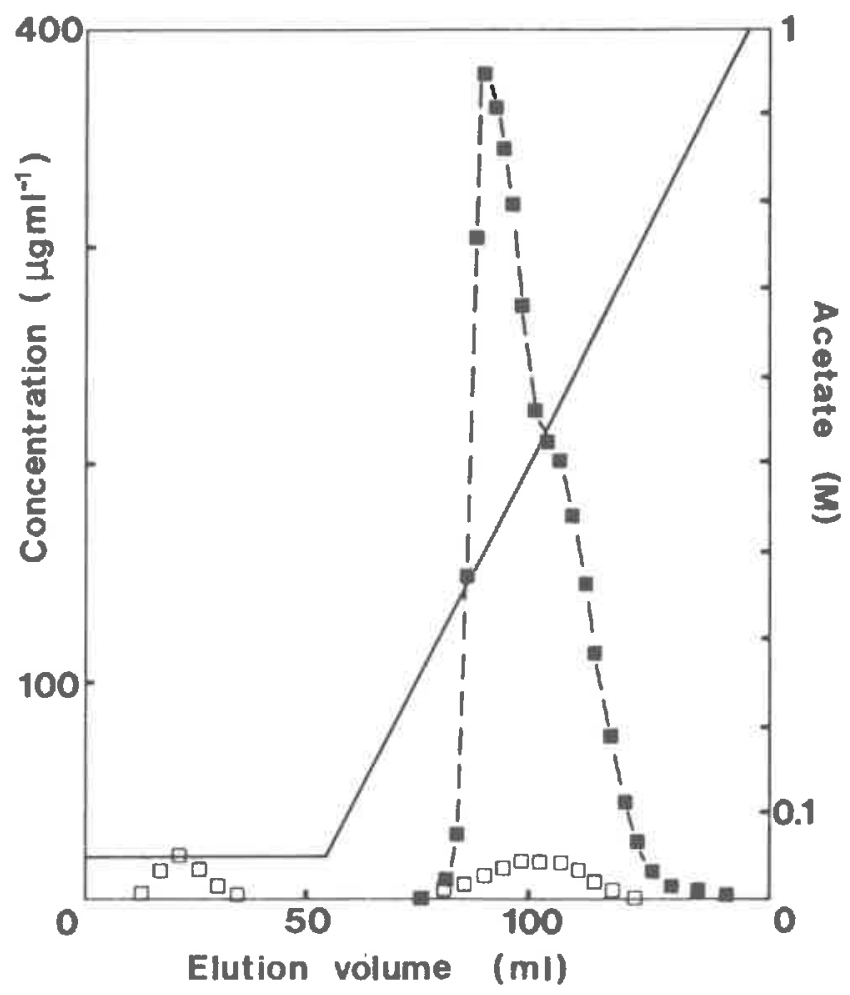

Figure 1 Ion-exchange chromatography of the crude $P_{72}$ citrus pectin on DEAE-Sepharose-CL-64. $\square$, Neutral sugars; w, galacturonic acids 
Table 1 Chemical composition of the initial citrus pectin $\mathbf{P}_{72}$ (mol/100 mol of total sugars)

\begin{tabular}{lcc}
\hline & Crude pectin & Purified pectin \\
\hline Galacturonic acid & 90.0 & 94.0 \\
Rhamnose & 1.0 & 0.7 \\
Arabinose & 3.3 & 1.8 \\
Xylose & 0.1 & 0.1 \\
Mannose & 0.2 & - \\
Galactose & 4.8 & 3.2 \\
Glucose & 0.6 & 0.2 \\
Degree of methylation & 71.4 & 72.0 \\
Degree of acetylation & $<1.0$ & $<1.0$
\end{tabular}

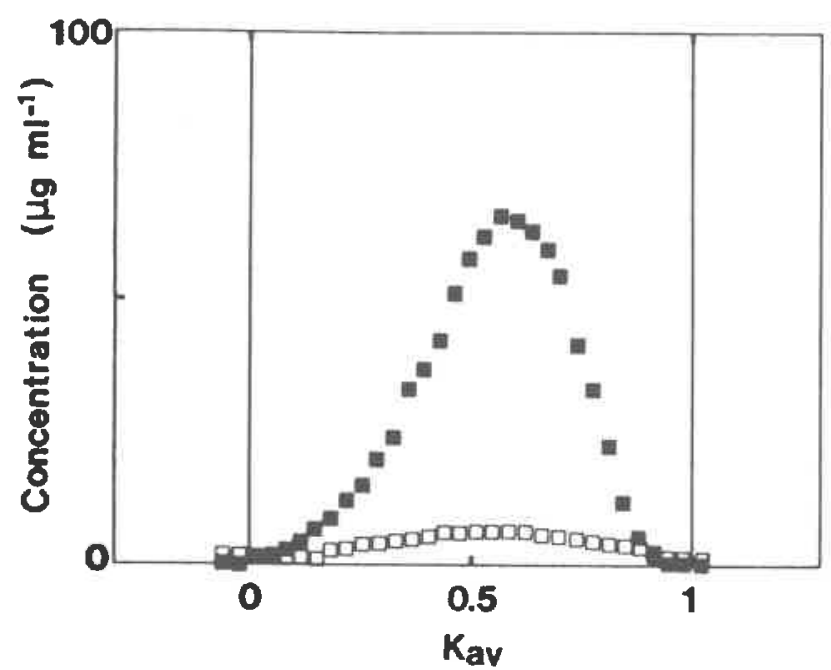

Figure 2 Gel permeation chromatography of the purified $P_{72}$ citrus pectin on Sepharose-CL-2B. $\square$, Neutral sugars; E, galacturonic acids

hydrodynamic volume distribution. At the maximum of the peak $\left(K_{\mathrm{av}}=0.6\right)$ the ratio AGA/neutral sugars is $13 / 1$ whereas the material with the highest hydrodynamic volume appears to be particularly rich in neutral sugars, with a ratio $0.9 / 1$.

The enzymatic degradation of the purified $P_{72}$ pectin after complete de-esterification leads to a percentage of hydrolysis of $41 \%$. The gel permeation chromatography of the products shows the presence of two populations (Figure 3): a high molecular weight fraction eluted in the void volume which contains all the neutral sugars and only $4.5 \%$ of total AGA, and a low molecular weight fraction eluted at the total volume which contains almost pure oligogalacturonides accounting for $95.5 \%$ of total AGA.

Since at least $95.5 \%$ of the galacturonic acid residues are obtained in a pure form and since enzyme cannot hydrolyse trigalacturonic acid ${ }^{15}$, these results show that the neutral sugars are not regularly distributed along the pectic backbone. So, the citrus pectins consist of long homogalacturonan regions and of some 'hairy' regions in which neutral sugars form side chains attached mainly on the rhamnose kinks ${ }^{2}$. As the rhamnose residues represent only $1 \%$, the overall conformation of the pectic molecules can be considered to be governed mainly by the DM and the distribution of methoxyl groups. Similar results have been obtained by enzymic degradation of citrus and apple pectins $^{16}$. This structural model appears to be in contradiction with that of Rees and co-workers in which pectins are represented as segmented rods with flexibility at the rhamnose kinks ${ }^{17,18}$.

Samples of pectins of various DM $\left(\mathrm{P}_{64}, \mathrm{P}_{53}, \mathrm{P}_{45}, \mathrm{P}_{38}\right.$, $P_{30}$ ) were obtained by alkaline de-esterification. This de-esterification process has been used to obtain samples with a random distribution of the carboxyl groups ${ }^{8}$. Their main characteristics (DM, AGA content and structural charge parameter $\lambda$ ) are indicated in Table 2. $\lambda$ is a fundamental parameter which represents the dimensionless charge density of the polyelectrolyte:

$$
\lambda=\left(e^{2} / D k T\right) /(100 b /(100-\mathrm{DM}))
$$

The numerator is the Bjerrum length, $L_{\mathbf{b}}=7.13 \AA$ in water at $25^{\circ} \mathrm{C}$, and the denominator is the average spacing between charges assuming a statistical repartition. $b$ is the length of the monomeric unit taken as $4.35 \AA^{17}$, $e$ is the electron charge, $D$ the dielectric constant of the solvent and $k T$ the Boltzmann term.

\section{Intrinsic viscosity and interaction parameters}

The intrinsic viscosity $[\eta]$ and the interaction parameters $\left(k_{1}, k_{2}\right)$ of the different sodium pectinate samples in $0.1 \mathrm{M} \mathrm{NaCl}$ solutions are determined in the specific viscosity range $0.2-1.5$ by the double extrapolation procedure based on the Huggins (equation 3) and Kraemer (equation 4) equations:

$$
\begin{aligned}
& \eta_{\mathrm{sp}} / C=[\eta]+k_{1}[\eta]^{2} C \\
& \left(\ln \eta_{\mathrm{r}}\right) / C=[\eta]+k_{2}[\eta]^{2} C
\end{aligned}
$$

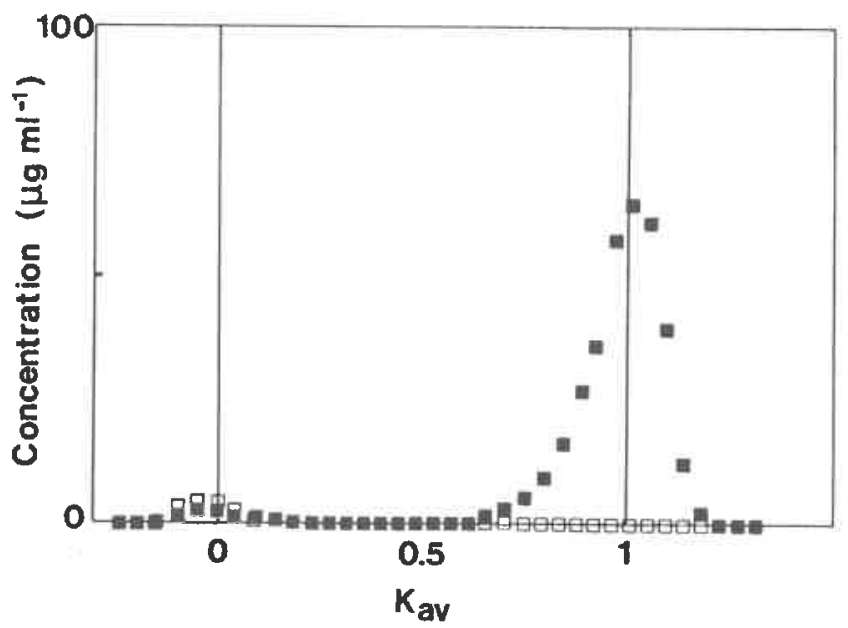

Figure 3 Gel permeation chromatography on SephacrylS-200 of the products obtained after enzymic degradation of the purfied $\mathrm{P}_{72}$ citrus pectin previously de-esterified. $\square$, Neutral sugars; $\mathbf{m}$, galacturonic acids

Table 2 Physicochemical parameters of the pectin samples

\begin{tabular}{lcccccc}
\hline & $\mathbf{P}_{72}$ & $\mathbf{P}_{64}$ & $\mathbf{P}_{53}$ & $\mathbf{P}_{45}$ & $\mathbf{P}_{38}$ & $\mathbf{P}_{30}$ \\
\hline AGA & 76 & 78 & 78 & 79 & 79 & 80 \\
$\mathrm{DM}$ & 72 & 64 & 53 & 45 & 38 & 30 \\
$\lambda$ & 0.451 & 0.579 & 0.757 & 0.886 & 0.998 & 1.127 \\
$\gamma_{\text {th }}$ & 0.798 & 0.749 & 0.685 & 0.642 & 0.607 & 0.538 \\
{$[\eta](1 / g)$} & 0.596 & 0.536 & 0.479 & 0.430 & 0.409 & 0.382 \\
$k_{1}$ & 0.55 & 0.45 & 0.37 & 0.47 & 0.52 & 0.59 \\
$k_{2}$ & 0.05 & 0.06 & 0.14 & 0.04 & 0.03 & 0.10 \\
\hline
\end{tabular}




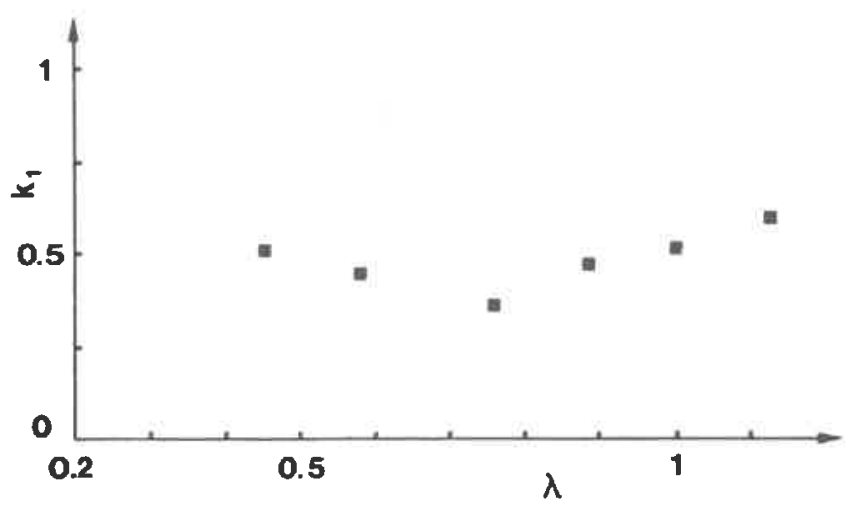

Figure 4 Evolution of the Huggins coefficient $k_{1}$ with the charge parameter $\lambda$

where $\eta_{\mathrm{sp}}$ is the specific viscosity $\left(\eta_{\mathrm{sp}} / C=\eta_{\mathrm{r}}-1\right), \eta_{\mathrm{r}}$ is the relative viscosity $\left(\eta_{\mathrm{r}}=\eta_{0} / \eta_{\mathrm{s}}, \eta_{\mathrm{o}}\right.$ is the viscosity of the solution extrapolated to zero shear-rate and $\eta_{s}$ is the solvent viscosity).

For all the samples, equations (3) and.(4) give linear plots leading to the same values of intrinsic viscosities with $k_{1}+k_{2}$ around the theoretical value of 0.5 ; the experimental precision is $\pm 3 \%$ on $[\eta]$ and $\pm 6 \%$ on $k_{1}$. Our results for $[\eta], k_{1}$ and $k_{2}$ are listed in Table 2. The steady decrease of $[\eta]$ from $0.596\left(\mathbf{P}_{72}\right)$ to $0.382 \mathrm{l} / \mathrm{g}\left(\mathbf{P}_{30}\right)$ may be due to some $\beta$-elimination reactions during the alkaline de-esterification despite the low temperature.

$k_{1}$ is plotted versus $\lambda$ in Figure 4 ; a minimum value is observed for charge parameter around 0.75 which corresponds to a DM of about $50 \%$. It is difficult to give an exact meaning to this minimum because the Huggins coefficient which characterizes the effect of the molecular interactions on the viscosity, exhibits a complex dependence on the energy and entropy of interaction between the polymer and the solvent. Experimental results obtained on a lot of polymers in different solvent have shown that an increase in $k_{1}$ indicates either a chain stiffening or an increase in the energy of intermolecular interactions ${ }^{19}$. At low DM, the increase in $k_{1}$ may be therefore explained by a chain stiffening due to the contribution of the electrostatic forces whereas the increase in $k_{1}$ at high DM may be interpreted by specific attractions between the methyl groups; the exact nature of these attractions is not well known. However, the changes of $k_{1}$ with DM are not very pronounced because of the high rigidity of the glycosidic bond which severely limits the relative orientations of adjacent galacturonic residues ${ }^{20}$.

\section{Concentration dependence of zero-shear viscosity}

The concentration dependence of the specific viscosity, obtained for zero shear rate, has been determined for each sodium pectinate sample keeping the ionic strength of the solutions constant for different dilutions. For a polyelectrolyte solution with added salt, the total ionic strength $(I)$ is the sum of the contribution of the salt $\left(I_{\mathrm{s}}\right)$ and of the polymer:

$$
I=I_{\mathrm{s}}+\gamma \alpha C
$$

where $C(\mathrm{eq} / \mathrm{l})$ is the polymer concentration, $\alpha$ the degree of dissociation and $\gamma$ the activity coefficient. In our case, $\alpha$ is one and the Manning's theory ${ }^{21}$ has been used to calculate $\gamma$ (Table 2) because it has been previously shown ${ }^{8}$ on similar pectins, that there is a good agreement between experimental and theoretical values.

Figure 5 justifies the use of the iso-ionic dilution procedure. For concentration higher than $5 \mathrm{~g} / \mathrm{l}$, significantly different curves are obtained by iso-ionic dilution and by dilution of the same pectin by $0.1 \mathrm{M} \mathrm{NaCl}$, showing the influence of the pectic charge groups on the ionic strength of the solution.

The dependence of the specific viscosity on concentration for all our samples has been compared using the dimensionless parameter $C[\eta]$ which measures the volume filling of a solution by a polymer ${ }^{19}$. The master curve thus obtained in a log-log plot (Figure 6) exhibits the classic behaviour ${ }^{22}$ with the transitions from dilute to semi-dilute solution at concentration $C^{*}$, and from semidilute to concentrated solution at another critical concentration $C^{* *} \gg C^{*}$. Up to $C[\eta]=C^{*}[\eta] \simeq 0.7$, specific viscosity scales as $C[\eta]^{x}$ with $x=1.2$ for all our samples. Above $C^{*}$ the gradient increases and reaches a constant value beyond $C[\eta]>C^{* *}[\eta] \simeq 8$ with $x=3.3$ which is in

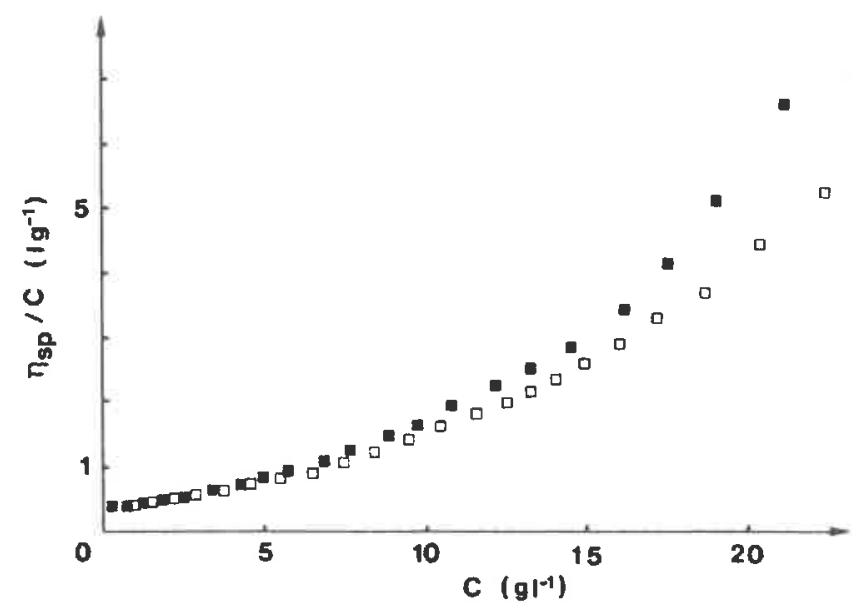

Figure 5 Influence of the dilution procedure on the viscosity of pectins. $\square$, Specific viscosity of the $P_{38}$ citrus pectin obtained by dilution by $0.1 \mathrm{M} \mathrm{NaCl}$. $\mathrm{m}$, Specific viscosity of the same pectin using iso-ionic dilution procedure

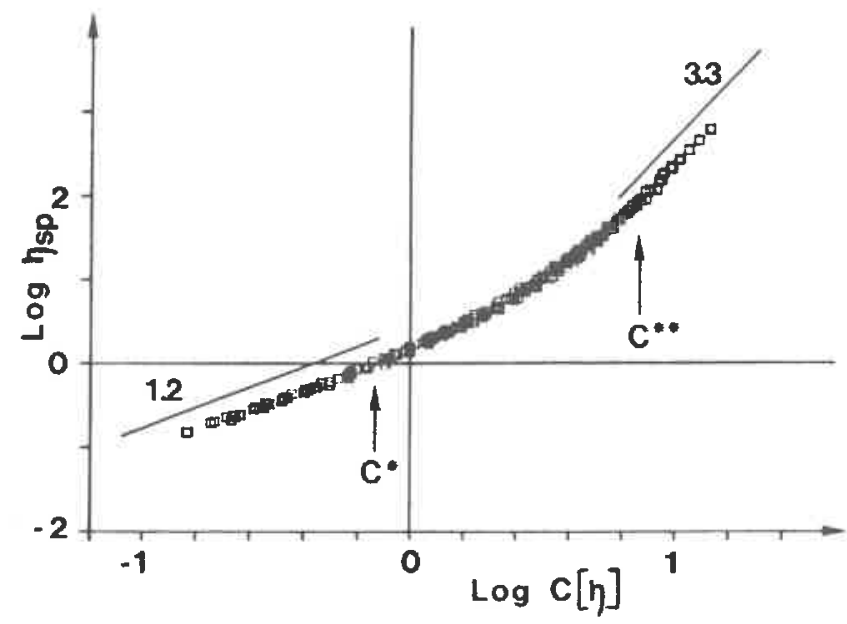

Figure $6 \eta_{\mathrm{sp}}$ versus $C[\eta]$ curve in a $\log -\log$ plot for all the citrus pectin samples with DM range between 30 and $72 \%$. $C^{*}$ is the overlap concentration between dilute and semi-dilute regimes; $C^{* *}$ is the boundary concentration between semi-dilute and concentrated regions 
good agreement with the expected 3.4 value for linear polymers of high molecular weight ${ }^{23}$.

At concentrations up to $C^{*}$, the average dimension of the coil is the same as for infinite dilution because the chain dimension is not perturbed by the other polymer molecules. An increase in $C$ above $C^{*}$ brings about an overlapping between the chains and a decrease in chain dimensions so that the unperturbed dimensions are approached. $C^{* *}$ has being defined as the concentration where chain dimensions become independent of $C$, tending to the $\theta$-state. The values of $\mathrm{C}^{*}$ and $C^{* *}$ allows us to approximate the expansion ratio of the chains at infinite dilution $\alpha_{0}^{22}$ :

$$
C^{* *}=C^{*}\left(\alpha_{0}\right)^{8}
$$

As $C^{* *} / C^{*}$ is $10, \alpha_{0}$ is estimated to be 1.33 . This value indicates that $0.1 \mathrm{M} \mathrm{NaCl}$ at neutral $\mathrm{pH}, 25^{\circ} \mathrm{C}$, is a good solvent for sodium pectinates, whatever the DM between 30 and $72 \%$.

\section{Determination of the Mark-Houwink exponent}

In the semi-dilute region, $d\left(\ln \eta_{\mathrm{r}}\right) / \mathrm{d} C$ is a decreasing function of $C$; this function, introduced by Simha and coworkers $^{24}$, may be related to the intrinsic viscosity at finite dilution. Assuming that entanglements do not significantly contribute to the viscosity for concentrations at the beginning of the semi-dilute regime ${ }^{22}$ the decrease of $\mathrm{d}\left(\ln \eta_{\mathrm{r}}\right) / \mathrm{d} c$ reflects only the contraction of the coil volume by osmotic compression. Under these conditions, using the expression for the polymer coil expansion coefficient established by Graessley ${ }^{22}$ and the Mark-Houwink relation, Lefebvre ${ }^{25}$ has given the following equation for the relative viscosity in the semidilute regime:

$$
\ln \left(\eta_{r}\right)=2 a[\eta] C^{*}\left(C / C^{*}\right)^{1 / 2 a}-(2 a-1) C^{*}[\eta]
$$

where $a$ is the Mark-Houwink exponent.

Figure 7 shows the experimental points for the $\mathrm{P}_{38}$ sample and the curve resulting from the fitting taking $C^{*}$ and $a$ as adjustable parameters. Equation (9) fits the experimental results well, the values for $a$ (Table 3 ) vary between 0.76 and 0.88 depending on the sample; these results confirm that $0.1 \mathrm{M} \mathrm{NaCl}$ is a good solvent of our polymers. The values of $C^{*}[\eta]$ obtained by the present procedure (Table 3 ) are in good agreement with the value determined from the master curve of Figure 6.

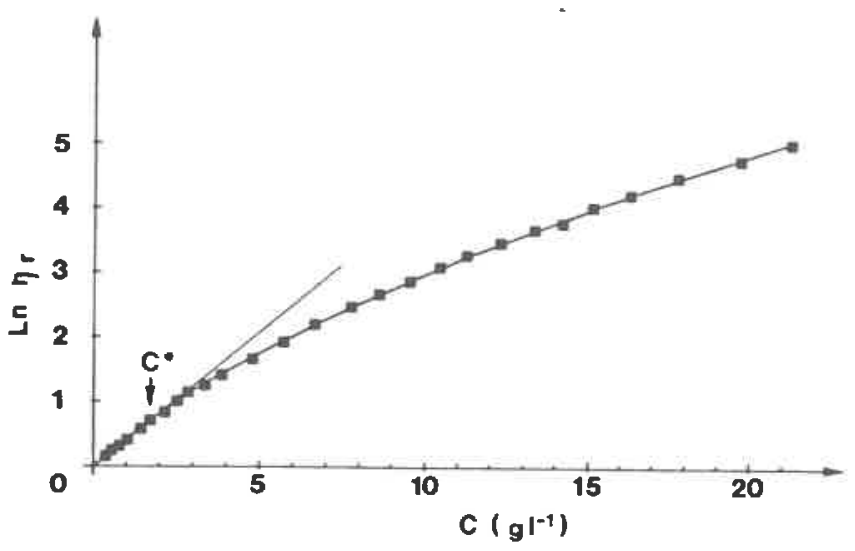

Figure 7 Plot of the relative viscosity versus concentration in a log-normal plot. ㅍ. Experimental points; — fitting obtained by using equation (9)
Table 3 Values of $C^{*}$ and Mark-Houwink exponents obtained from the fit of the experimental points $\ln \eta_{\mathrm{r}}$ versus $C$ (Figure 7)

\begin{tabular}{llllll}
\hline & $P_{72}$ & $P_{53}$ & $P_{45}$ & $P_{38}$ & $P_{30}$ \\
\hline$C^{*}(g / 1)$ & 1.27 & 1.46 & 1.88 & 1.70 & 1.67 \\
$a$ & 0.81 & 0.76 & 0.88 & 0.80 & 0.80 \\
$c^{*}[\eta]$ & 0.76 & 0.70 & 0.81 & 0.69 & 0.64
\end{tabular}

The values of $a$ are very similar to those $(a=0.73)$ recently determined by Anger and Berth ${ }^{5}$ for a set of sodium pectinates. This value is far from the value (1.34) given previously by Owens et al. ${ }^{26}$ which suggested that pectins are very rigid polymers. This discrepancy apparently may be ascribed to badly-defined samples used by Owens et al., as previously discussed by Anger and Berth ${ }^{5}$. Value of $a$ higher than 1 has been reported only for very stiff molecules such as xanthan ${ }^{27}$.

Conformational features obtained by Burton and Brant indicate also that 1-4 linked $\alpha$ galactans generate a very stiff polymer ${ }^{20}$. These results cannot be taken as an absolute prediction of the real pectins conformation in solution ${ }^{20}$ but could indicate that the homogalacturonan region is rigid. The relative flexibility obtained here from hydrodynamic experiments could arise (1) from the random insertions of 'hairy' regions on the polygalacturonic backbone which can reduce considerably the extended nature of the chains, (2) from additional intramolecular hydrogen bonding between neutral sugars and/or methoxyl groups but such associations have not been experimentally proved. In our opinion, definitive conclusion concerning the chain flexibility may be obtained from the direct measurement of the persistence length by small-angle $X$-ray or neutron scattering because these methods are less sensitive to aggregation and molecular weight distribution ${ }^{28}$.

\section{Conclusion}

From the study of the flow behaviour of citrus pectins an expansion ratio of 1.33 and a Mark-Houwink exponent of 0.8 were found; it can be concluded that $0.1 \mathrm{M} \mathrm{NaCl}$, $25^{\circ} \mathrm{C}$, is a good solvent for sodium pectinates whatever the DM between 30 and $72 \%$. These results also indicate that pectins appear as relatively flexible polymers. More generally the concentration dependence of the specific viscosity of all our pectin samples follows the general behaviour of linear and flexible polymers in good solvent ${ }^{29.30}$. The variation of the Huggins parameter with DM indicates that a maximum of flexibility occurs for an intermediate DM of $50 \%$ in agreement with previous results obtained by light scattering ${ }^{4}$.

\section{Acknowledgements}

The authors are grateful to Dr S. Perez for fruitful discussions. We would also like to thank Dr M. Kolb for a critical reading of the manuscript and to Mrs M. J. Crepeau and Mr M. Branger for technical assistance.

\section{References}

1 Nelson, D. B., Smit, C. J. B. and Wiles, R. R. in 'Food Colloids' (Ed. H. D. Graham), Avi, Westport, CT, 1977, p. 418 
2 Darvill, A., MacNeil, M., Albersheim, P. and Delmer, D. P. in 'The Biochemistry of Plants' (Ed. N. E. Colbert), Academic Press, New York, 1980, Vol. 1, p. 91.

3 Fishman, M. L., Pfeffer, P. E., Barford, R. A. and Doner, L. W. J. Agric. Food Chem. 1984, 32, 372

4 Plashchina, I. G., Semenova, M. G., Braudo, E. E. and Tolstoguzov, V. B. Carbohydr. Polym. 1985, 5, 159

5 Anger, H. and Berth, G. Carbohydr. Polym. 1986, 6, 193

6 Hourdet, D. and Muller, G. Carbohydr. Polym. 1987, 7, 301

7 Michel, F., Thibault, J. F. and Doublier, J. L. Sci. Alim. 1981, 4, 569

8 Thibault, J. F. and Rinaudo, M. Biopolymers 1985, 24, 2131

9 Thibault, J. F. and Mercier, C. J. Solid-Phase Biochem. 1977, 2, 295

Nelson, N. J. Biol. Chem. 1944, 153, 375

Thibault, J. F. Lebensm. Wiss. U. Technol. 1979, 12, 247

Voragen, A. G. J., Schols, H. A. and Pilnik, W. Food Hydrocolloids 1986, 1, 65

13 Blakeney, A. B., Harris, P. J., Henry, R. J. and Stone, B. A. Carbohydr. Res. 1983, 113, 291

Tollier, M. T. and Robin, J. P. Ann. Technol. Agric. 1979, 28,

Thibault, J. F. and Mercier, C. J. Food Biochem. 1978, 2, 379

De Vries, J. A., Rombouts, F. M., Voragen, A. G. J. and Pilnik, W. Carbohydr. Polym. 1984, 4, 89
Rees, D. A. and Wight, A. W. J. Chem. Soc. B 1971, 1366 Powell, D. A., Morris, E. R., Gidley, M. J. and Rees, D. A J. Mol. Biol. 1982, 155, 517

Dreval, V. E., Malkin, A. Ya. and Botvinnik, G. O. J.Polym.Sci. $1973,11,1055$

Burton, B. A. and Brant, D. A. Biopolymers 1983, 22, 1769

Mannings, G. S. J. Chem. Phys. 1969, 51, 924

Graessley, W. W. Polymer 1980, 21, 258

Bohdanecky, M. and Kovar, J. in "Viscosity of Polymer Solutions’ (Ed. A. D. Jenkins), Elsevier Scientific, Amsterdam, 1982, p. 193

4 Weissberg, S. G., Simha, R. and Rothman, S. J. Res. Nat. Bureau Standards 1951, 47, 298

Lefebvre, J. Rheol. Acta 1982, 21, 620

6 Owens, H. S., Lotzkar, H., Schultz, T. H. and Maclay, W. D. J. Am. Chem. Soc. 1946, 68, 1628

Milas, M., Rinaudo, M. and Tinland, B. Polym. Bull. 1985, 14, 157

Axelos, M. A. V., Lefebvre, J. and Thibault, J. F. Food Hydrocolloids 1987, 1, 570

Morris, E. R., Cutler, A. N., Ross-Murphy, S. B. and Rees, D. A. Carbohydr. Polym. 1981, 1, 5

Castelain, C., Doublier, J. L. and Lefebvre, J. Carbohydr. Polym. $1987,7,1$ 